\title{
PAPER
}

\section{Influence of clinical, demographic, and socioeconomic variables on quality of life in patients with epilepsy: findings from Georgian study}

\author{
M Diibuti, R Shakarishvili
}

See end of article for authors' affiliations

.................

Correspondence to: Dr M Dibuti, Department of Public Health and Epidemiology, State Medical Academy, 380079 Tbilisi, Georgia; m.djibuti@curatio.com

Received 10 September 2002

Accepted

11 January 2003

\begin{abstract}
Objective: To identify the clinical, demographic, and socioeconomic factors that are associated with a poor quality of life in patients with epilepsy in Georgia.

Methods: Clinical, demographic, and socioeconomic status data were collected from 115 adult epileptic outpatients being treated in the epilepsy programme at the Sarajishvili Institute of Neurology and Neurosurgery (SINN) in Tbilisi, Georgia. Health Related Quality of Life (HRQL) was measured by the Quality of Life in Epilepsy Inventory (QOLIE-31). Multiple regression analysis was used to determine which variables were associated with QOLIE-31 total and subcomponent scores.

Results: Mean age of the patient population was 37.9 (SD 15.8) years; $43.5 \%$ were females; $51.8 \%$ did not have a partner; $39.1 \%$ had some university education; $82.6 \%$ were unemployed. Of 115 epileptic patients $83.3 \%$ had partial, and $16.7 \%$ had generalised seizures. Overall, $32.2 \%$ of patients were seizure free, and $28.7 \%$ experienced more than 10 seizures over the past year. The variables that most strongly predicted a lower QOLIE-31 total score were a low education level, high seizure frequency, and long duration of epilepsy. The QOLIE-31 all subcomponent scores correlated strongly with seizure frequency. Advanced age was a significant predictor for a low overall quality of life, energy/fatigue, and cognitive scores. Female sex was the factor that significantly predicted a low seizure worry score. Education level strongly correlated with overall quality of life, and cognitive and social functioning scores.

Conclusions: Clinical factors such as high seizure frequency and long duration of epilepsy had a significant influence on HRQL. Advanced age, female sex, and a low education level were the demographic factors that correlated strongly with low quality of life scores.
\end{abstract}

$\mathrm{H}$ ealth related quality of life (HRQL) has been reported to vary across epileptic patients with different clinical, demographic, and socioeconomic variables. ${ }^{1-9}$ With regard to clinical variables, seizure type and frequency have been found to be significant predictors of HRQL scores. ${ }^{1{ }^{3}}$ The role of demographic variables such as age, sex, and education, as well as socioeconomic status (SES) in determining HRQL is less clear. In some studies age, sex, and education did not correlate significantly with the HRQL. ${ }^{56}$ Several studies have highlighted that patients with epilepsy are more likely to be underemployed or unemployed, and have lower rates of marriage. ${ }^{278}$

This information has come mainly from developed countries, ${ }^{13-9}$ whereas, to our knowledge, there is only one study that originates from a former Soviet country-Estonia. ${ }^{2}$ Sociodemographic factors may exert different effects on HRQL in patients with epilepsy in former Soviet countries, which currently face problems of political, social, and economic transition. The purpose of the present study was to examine the impact of different clinical, demographic, and SES variables on the HRQL in a non-surgical sample of adults with epilepsy in Georgia, one of the former Soviet countries.

\section{METHODS}

\section{Participants, recruitment, and data collection}

The study recruited respondents at the Sarajishvili Institute of Neurology and Neurosurgery (SINN), which is a national leading centre providing care for patients suffering from epilepsy in Georgia. Since 1995, the SINN has been implementing an epilepsy treatment programme supported by international humanitarian organisations, which has been providing the patients with free antiepileptic drugs (AED).
Every month, patients were taking a variety of AEDs, including carbamazepine, divalproex sodium, phenobarbital, and phenytoin. The sampling frame consisted of 1073 patients satisfying the following criteria: 18 years of age or older; diagnosis of epilepsy for a minimum of one year; and prescribed AED at the time of recruitment. Patients were excluded from the sampling frame if they had serious physical or mental limitation not allowing them to complete the Quality of Life in Epilepsy Inventory (QOLIE-31) questionnare. In addition, patients with psychiatric or neurological disorders that would impair judgement or impact quality of life beyond the effects caused by epilepsy (including but not limited to dementia, mental retardation, stroke, head injury, brain tumour, cerebral palsy, and current clinical depression) or who had undergone any form of epilepsy surgery were excluded. Of these 1073 patients satisfying the aforementioned criteria and being enrolled into the epilepsy treatment programme by September 1999, 150 patients were selected by simple random sampling as participants of the present study.

All selected patients were entered into the study between September 1999 and February 2000. A patient's consultation at the SINN was used to identify and record clinical, demographic, and socioeconomic data, and to provide him/her with the quality of life questionnaire, which had to be completed at the patient's home and returned to SINN during a subsequent visit within one month. The extent of

Abbreviations: AED, antiepileptic drugs; $H R Q L$, health related quality of life; QOLIE-31, Quality of Life in Epilepsy Inventory; SES, socioeconomic status; SINN, Sarajishvili Institute of Neurology and Neurosurgery 
Table 1 Clinical, demographic, and socioeconomic characteristics of the patient population

\begin{tabular}{ll}
\hline Age (mean years (SD)) & 37.9 (15.8) \\
Sex (\% female) & 43.5 \\
Married/cohabitating (\%) & 48.2 \\
Education level (\%) & \\
$\quad$ Primary/secondary/technical/vocational & 60.9 \\
$\quad$ University studies & 39.1 \\
Unemployed (\%) & 82.6 \\
Permanent access to a car and/or mobile phone (\%) & 19.1 \\
Epileptic seizure type (\%) & 83.3 \\
$\quad$ Partial seizures & 16.7 \\
$\quad$ Generalised seizures & \\
Seizure frequency over the past year (\%) & 32.2 \\
$\quad$ Free of seizures & 39.1 \\
$\quad<10$ & 28.7 \\
$\quad \geqslant 10$ & 18.7 (12.5) \\
Duration of epilepsy (mean years (SD)) &
\end{tabular}

completion of the study questionnaire was checked on return, and patients were asked to complete any missing sections. Thus, there were no unscorable questionnaires. All patients gave their informed consent for participation in the research.

\section{Study variables}

We recorded a number of clinical, demographic, and socioeconomic variables with possible prognostic influence on HRQL. Epileptic seizures were classified by the Revised Clinical and Electroencephalographic Classification of Epileptic Seizures. ${ }^{10}$ For the purpose of analysis, the patients were categorised into two groups: (1) patients with partial seizures (simple partial seizures, complex partial seizures, and partial seizures evolving to secondary generalised seizures); and (2) those with generalised seizures (absence, atypical absence, myoclonic seizures, clonic, tonic, tonic-clonic, and atonic seizures).

According to the number of seizures over the past year, the patients were divided into three categories: free of seizures, less than 10 seizures, and 10 or more seizures. To be classified as seizure free, patients had to have been seizure free for at least the year before inclusion into the study. We also recorded duration of epilepsy. For all patients, we recorded age, sex, education level, and whether they lived with or without a partner. For evaluation of the patient's socioeconomic position, the study collected the data on employment status and permanent access to a car and/or mobile phone (as proxy measures of income).

The HRQL was measured by the Quality of Life in Epilepsy Inventory (QOLIE-31), which has been extensively tested for validity and reliability. ${ }^{11}$ The QOLIE-31 measures HRQL across seven domains such as seizure worry, overall quality of life, emotional wellbeing, energy-fatigue, cognitive functioning, medication effects, and social functioning. The translation into Georgian was done according to international principles (series of forward and backward translations followed by reconciliation discussion by English language and epilepsy experts). The translated questionnaire was pretested in five Georgian patients in order to assess clarity, appropriateness of wording, understandability, and cultural relevance of the translated version.

\section{Data analysis}

We carried out multiple regression analysis to explore the association between the possible prognostic variables and reduced QOLIE-31 total and subcomponent scores using SPSS for Windows (version 7.0; SPSS, Inc., Chicago, IL). In addition, we tested for interaction effects of second degree product terms of sex, marital/cohabitating status, education level, seizure frequency, and duration of epilepsy. None of these interaction effects were found significant at the $5 \%$ level. Thus, the final models included main effects only. For the assessment of reliability of the Georgian version of QOLIE-31, we calculated internal consistency reliability coefficients (Chronbach's $\alpha$ ) for each scale.

\section{RESULTS}

\section{Sample characteristics}

Of 150 patients initially selected for the study, the HRQL questionnaire was completed and returned by 115 (76.7\%) patients. The mean age of the respondents was 37.9 (SD 15.8) years. There was no significant difference between respondents and non-respondents in terms of the main clinical and

Table 2 Information on the strongest predictors for six subscores

\begin{tabular}{|c|c|c|c|c|c|c|}
\hline Variable & $\begin{array}{l}\text { Univariate regr } \\
\text { coeff }\end{array}$ & $\begin{array}{l}\text { Multivariate regr } \\
\text { coeff }\end{array}$ & SEM & $t$ value & $p$ value & Adjusted $\mathrm{R}^{2}$ \\
\hline \multicolumn{7}{|l|}{ QOLIE-31 total score } \\
\hline Education & 19.84 & 0.28 & 6.83 & 2.82 & 0.005 & \multirow[t]{3}{*}{0.23} \\
\hline Seizure frequency $\geqslant 10$ & -22.34 & -0.29 & 8.08 & -2.77 & 0.007 & \\
\hline Duration of epilepsy & 0.64 & 0.23 & 0.25 & 2.55 & 0.012 & \\
\hline \multicolumn{7}{|l|}{ Seizure worry } \\
\hline Sex & 10.74 & 0.19 & 5.35 & 2.01 & 0.048 & \multirow[t]{3}{*}{0.15} \\
\hline Seizure frequency $\geqslant 10$ & -13.12 & -0.21 & 6.61 & -1.98 & 0.050 & \\
\hline Duration of epilepsy & 0.55 & 0.25 & 0.25 & 2.19 & 0.003 & \\
\hline \multicolumn{7}{|l|}{ Overall quality of life } \\
\hline Age & -0.34 & -0.30 & 0.11 & -3.03 & 0.003 & \multirow[t]{3}{*}{0.20} \\
\hline Education & 7.85 & 0.22 & 3.41 & 2.30 & 0.023 & \\
\hline Seizure frequency $\geqslant 10$ & -8.77 & -0.23 & 4.05 & -2.16 & 0.033 & \\
\hline \multicolumn{7}{|l|}{ Emotional wellbeing } \\
\hline Seizure frequency $\geqslant 10$ & -9.39 & -0.23 & 4.60 & -2.04 & 0.044 & 0.10 \\
\hline \multicolumn{7}{|l|}{ Energy/fatique } \\
\hline Age & -0.30 & -0.25 & 0.12 & -2.47 & 0.015 & \multirow[t]{2}{*}{0.19} \\
\hline Seizure frequency $\geqslant 10$ & -10.14 & -0.24 & 4.45 & -2.28 & 0.025 & \\
\hline \multicolumn{7}{|l|}{ Cognitive functioning } \\
\hline Age & -0.44 & -0.29 & 0.15 & -2.99 & 0.004 & \multirow[t]{3}{*}{0.27} \\
\hline Education & 17.19 & 0.34 & 4.57 & 3.76 & 0.000 & \\
\hline Seizure frequency $\geqslant 10$ & -16.72 & -0.31 & 5.44 & -3.08 & 0.003 & \\
\hline \multicolumn{7}{|l|}{ Social functioning } \\
\hline Education & 15.11 & 0.27 & 5.30 & 2.85 & 0.005 & \multirow[t]{3}{*}{0.18} \\
\hline Seizure frequency $\geqslant 10$ & -16.64 & -0.28 & 6.31 & -2.64 & 0.010 & \\
\hline Duration of epilepsy & 0.40 & 0.18 & 0.20 & 1.98 & 0.050 & \\
\hline
\end{tabular}


sociodemographic characteristics. Table 1 presents clinical, demographic, and socioeconomic characteristics of the patient population.

\section{Multiple regression analysis}

Variables from table 1 were included in the multiple regression analysis as independent variables. As dependent variables we entered QOLIE-31 total and seven subscores separately. Table 2 shows that lower education, higher seizure frequency, and longer history of disease were the variables that most strongly predicted lower QOLIE-31 total score. The full model explained $23 \%$ of the variance of the QOLIE-31 total score. Similarly, table 2 presents information on the strongest predictors for six subscores such as seizure worry, overall quality of life, emotional wellbeing, energy/fatigue, cognitive functioning, and social functioning. There were no variables significantly associated with medication effects score.

There was a weak correlation between age and duration of epilepsy (Pearson's $r=0.29, \mathrm{p}=0.001$ ). Age remained a significant predictor for overall quality of life, energy/fatigue, and cognitive scores, when duration of epilepsy was controlled for. Similarly, duration of epilepsy was an independent predictor for seizure worry score.

\section{Reliability of Georgian version of QOLIE-31}

The QOLIE-31 internal consistency reliability levels in this study comprised 0.78 (seizure worry), 0.74 (overall quality of life), 0.78 (emotional wellbeing), 0.75 (energy-fatigue), 0.82 (cognitive functioning), 0.71 (medication effects), and 0.74 (social functioning). These values suggest minimal measurement error associated with HRQL evaluation with the Georgian version of QOLIE-31.

\section{DISCUSSION}

The main focus of this study was to examine the effects of different clinical and sociodemographic factors on HRQL in patients with epilepsy in the former Soviet republic Georgia. We accept that one major methodological flow of this study is biased sampling: the patients with serious physical or mental limitations which prevented them from completing the QOLIE-31 questionnaire were excluded from the study. Therefore, the results may be biased towards the opinions of people with better general health status or higher education. Owing to the fact that SINN is a tertiary centre, one may consider extrapolation of the study findings to the general population of Georgia as misleading. In Georgia, patients with epilepsy are commonly referred to and treated in specialised institutions, for example SINN, which is a national leading centre for epilepsy. Furthermore, availability of free antiepileptic drugs should have helped inclusion of many patients with lower SES. Based on the above considerations, we believe that a sufficient amount of this data can be generalised to people with epilepsy in Georgia, although we recognise that the results of this study should be interpreted with some caution.

In this study, high seizure frequency had a significant influence on all domains of HRQL. This is in line with the results of other studies proving seizure frequency to be a significant inverse predictor of HRQL across different domains. ${ }^{1-4}$ This study also found that a long duration of disease has a significant positive impact on QOLIE-31 total score as well as seizure worry and social functioning. This may indicate that patients with longer anamnesis of epilepsy are better adjusted to the psychosocial consequences of their illness. Certainly, further research is needed to prove this statement.

An advanced age was significantly associated with low overall quality of life, energy-fatigue, and cognitive functioning. A significant association was also reported between age and SF-36 domain such as physical role limitations, bodily pain, and physical functioning scores in subjects with epilepsy in Estonia as well as 15 other European countries. ${ }^{2}{ }^{12}$ The study conducted in the UK has found that older people do not necessarily experience poorer quality of life than younger people, and only those first diagnosed in later life do appear to have a quality of life which is more impaired. ${ }^{5}$

In our study, female sex was a significant predictor for low seizure worry score. A similar result was found in the study conducted by Buck and colleagues, ${ }^{12}$ according to which females in 15 European countries had poorer scores on energy/vitality, mental health, general health, bodily pain, and physical functioning domains of SF-36. The fact that women had a worse HRQL in both developed and transitional countries may indicate that variations in the availability and accessibility of social and health care as well as socioeconomic factors are less important, and biological and/or psychological factors (for example, problems related with family, personal life, motherhood, etc) may play more critical role. In this connection, further research is needed to explain the reasons for poorer quality of life in female patients with epilepsy.

Very few patients with epilepsy (17\%) in our study sample were employed. In Estonia, it was found that the level of employment among persons with epilepsy (65\%) was not lower than that in the general population. ${ }^{2}$ This can be attributed to the fact that the Estonian study selected patients through a population based epidemiological survey, whereas our sample was composed of patients registered in a tertiary level clinic. In a large study that recruited patients from neurological practices in 15 European countries, reported proportion of the subjects with epilepsy in paid employment varied from as low as 32\% in Spain to as high as $65 \%$ in Sweden. ${ }^{12}$ In the same study, employment status was significantly associated with all eight domain scores of SF-36. In our study, however, this factor did not contribute significantly to lower quality of life scores.

In addition to clinical factors, this study revealed education as one of the strongest predictors of low HRQL. This was true for QOLIE-31 total, overall quality of life, and cognitive and social functioning scores. In studies on patient populations from the western world we could not find any study that assessed the role of education in determining HRQL. In western societies, different measures of SES are closely correlated: better educated people are employed and have higher income. Because of this close correlation between different indicators of SES, these studies have probably focused on employment only. The situation may be different in Georgia, where income levels of many people with high educational attainments were disrupted mainly because of macroeconomic changes. This lack of correlation between individual components of SES makes it reasonable to assess the role of education in determining HRQL. Taking into account the results of our study it can be concluded that in the environment where educational attainments were high and population employment levels were disrupted mainly because of macroeconomic changes, the education level seems to be a more significant determinant of HRQL in people with epilepsy.

\section{ACKNOWLEDGEMENTS}

We thank RAND for providing the QOLIE-31 questionnaire and for granting permission for using it in our study. The authors are also grateful to Drs R Nikolaishvili, O Toidze, T Chincharauli, L Sturua, S Kasradze, and A Karaulashvili, as well as the staff members of the outpatient department of SINN for providing support in implementation of the present study.

Authors' affiliations

M Diibuti, Department of Public Health and Epidemiology, State Medical Academy, 380079 Tbilisi, Georgia

R Shakarishvili, Sarajishvili Institute of Neurology and Neurosurgery,

380092 Tbilisi, Georgia

\section{REFERENCES}

1 Leidy NK, Elixhauser A, Vickrey B, et al. Seizure frequency and the health-related quality of life of adults with epilepsy. Neurology 1999;53:162-6. 
2 Herodes M, Oun A, Haldre S, et al. Epilepsy in Estonia: a quality-of-life study. Epilepsia 2001;42:1061-73.

3 Baker GA, Gagnon D, McNulty P. The relationship between seizure frequency, seizure type and quality of life: findings from three European countries. Epilepsy Res 1998;30:231-40.

4 Baker GA, Jacoby A, Buck D, et al. Quality of life of people with epilepsy: a European Study. Epilepsia 1997;38:353-62.

5 Baker GA, Jacoby A, Buck D, et al. The quality of life of older people with epilepsy: findings from a UK community study. Seizure $2001 ; 10: 92-9$

6 Suurmeijer TP, Reuvekamp MF, Aldenkamp BP. Social functioning, psychological functioning, and quality of life in epilepsy. Epilepsia 2001;42:1160-8.

7 Fraser RT, Clemmons D, Trejo W, et al. Program evaluation in epilepsy rehabilitation. Epilepsia 1983;24:734-46.
8 Hauser WA, Hesdorffer DC. Epilepsy: frequency, causes and consequences. Maryland: Epilepsy Foundation of America, 1990.

9 Jacoby A. Impact of epilepsy on employment status: findings from a UK study of people with well-controlled epilepsy. Epilepsy Res 1995;21:125-32

10 Commission on Classification and Terminology of the International League Against Epilepsy. Proposal for revised clinical and electroencephalographic classification of epileptic seizures. Epilepsia 1981;22:489-501.

11 Cramer JA, Perrine K, Devinsky O, et al. Development and cross-cultural translations of a 31 -item quality of life in epilepsy inventory. Epilepsia 1998;39:81-8

12 Buck D, Jacoby A, Baker GA, et al. Cross-cultural differences in health-related quality of life of people with epilepsy: findings from a European study. Qual Life Res 1999;8:675-85.

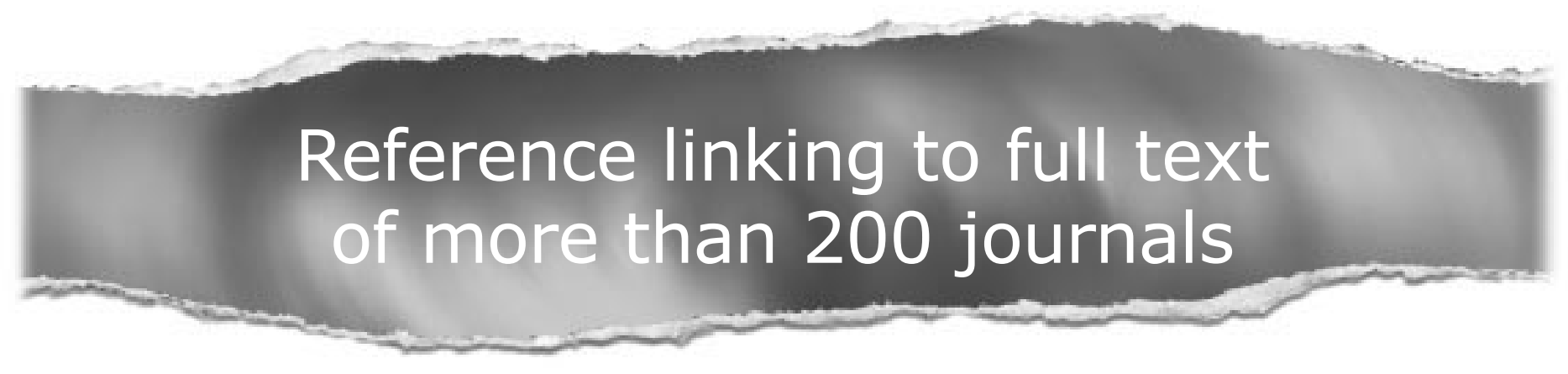

\section{Toll free links}

You can access the FULL TEXT of articles cited in the Journal of Neurology, Neurosurgery, and Psychiatry online if the citation is to one of the more than 200 journals hosted by HighWire (http://highwire.stanford.edu) without a subscription to that journal.

There are also direct links from references to the Medline abstract for other titles. 